\title{
PREDICTING THE RISK OF MYOCARDIAL INFARCTION USING DIFFERENT CLASSIFICATION ALGORITHMS
}

Fatemeh Rahimi ${ }^{1}$, Mahdi Nasiri ${ }^{2}$, Reza Safdari ${ }^{3}$, Roxana Sharifian ${ }^{4}$, Goli Arji ${ }^{5}$, Zahra khanom Hashemi ${ }^{6}$

1: Master student in Medical Informatics, Student Research Committee, Shiraz University of Medical Sciences (SUMS), Shiraz, Iran.

2: Mahdi Nasiri, school of management \& medical information sciences, Shiraz University of Medical Sciences (SUMS), Shiraz, Iran.

3: Professor in Health Information Management, Department of Health Information Management, Tehran University of Medical Sciences, Tehran, Iran.

4: Assistant Professor in Health Information Management, Shiraz University of Medical Sciences (SUMS), Shiraz, Iran.

5: Ph.D. Candidate in Health Information Management, Department of Health Information Management, Tehran University of Medical Sciences, Tehran, Iran.

6: Assistant professor of Pediatric Dentistry,dental school, Yasuj University of Medical Sciences ,Yasuj, Iran.

Correspondence:

Fatemeh Rahimi, Tel: +989396093135, E-mail: r.fatemeh70@yahoo.com

\section{TYPE OF ARTICLE: CONFERENCE ABSTRACT}

\begin{abstract}
Introduction: According to WHO, cardiovascular diseases (CVDs) are the leading cause of death globally. Although significant progress has been made in the diagnosis of CVDs, more investigation can be helpful. Considering the significant role of data mining techniques in discovering hidden trends in health data, the present study aimed to predict the risk of myocardial infarction (MI) using k-nearest neighbor (KNN), neural network, decision tree (C5), and Bayesian network algorithms.

Methods: The present study is an analytical study. Considering CRISP methodology, data-mining analysis was carried out using KNN, neural network, C5, and Bayesian network algorithms by IBM SPSS modeler (Clementine 14.2) software. The applied data were related to 350 patients admitted to the Shahid Rajaei specialized cardiovascular hospital. However, after cleaning the data, only 274 records were entered in final analysis.

Results: This study revealed that the highest percentages of correct classifications achieved were $67.42 \%$, $64.04 \%, 60.67 \%$, and $60.67 \%$ for the KNN, C5, neural network, and Bayesian network algorithms, respectively. The results also revealed that smoking was the leading risk factor for MI.

Conclusion: In terms of accuracy, KNN was the most effective algorithm in predicting MI, followed by C5, neural network, and Bayesian network.

KEYWORDS: KNN, Decision trees, C5, Neural network, Bayesian network, Myocardial infarction, Data mining.
\end{abstract}

\footnotetext{
Abstracts of First National Congress of Medical Informatics, Mashhad, Iran, February 2017

(C) 2017 The Authors. This is an open access article under the terms of the Creative Commons Attribution-NonCommercialNoDerivs License, which permits use and distribution in any medium, provided the original work is properly cited, the use is non-commercial and no modifications or adaptations are made.
} 